\title{
Assessing fungal risk criteria via simulated scenarios to address disparity between method and outcomes
}

\author{
Hannah, N. ${ }^{\mathrm{a}}$, Marincioni, V. ${ }^{\mathrm{a}}$, Chalabi, Z. ${ }^{\mathrm{a}}$, Altamirano-Medina, H. ${ }^{\mathrm{a}}$ \\ a UCL Institute for Environmental Design and Engineering, London, UK
}

\begin{abstract}
Internal wall insulation is one of the most promising methods of improving the energy efficiency of buildings while maintaining their original facades and construction. However, porous materials or poor construction quality can allow moisture ingress, creating favourable conditions for mould development within building fabric. Currently, there is no established guidance on moisture criteria in the construction industry for the assessment of interstitial mould growth. Some studies have considered relative humidity (RH) criteria that account for the duration of exposure but potentially overestimate risk at interfaces. This study implemented more specific RH criteria, based not only on the duration of exposure but also on temperature and substrate material. Results demonstrated a dramatic decrease in predicted mould risk, with minimal risk to health or structural integrity, in comparison to the present more stringent standards.

Peer-review under the responsibility of the organizing committee of the ICMB21.
\end{abstract}

Keywords: Internal Wall Insulation; Interstitial Condensation; Mould/Fungal Risk Assessment; Material/Insulation System Failure

\section{Introduction}

\subsection{Background}

Current UK Building Regulations set the threshold of material failure due to mould risk at 0\% [1]. However, according to recent research carried out by UCL all insulation materials used as internal wall insulation are at risk of mould development, exceeding the set failure criteria as often as $80 \%$ of the year based on data simulated on various wall constructions and weather scenarios across two locations in the UK [2]. The current guidance (BS 5250:2011 [3], Approved Document F (ADF) [1]) are based on assumptions of ideal conditions for mould growth, and a majority of the consultancy work is done considering $80 \%$ monthly averaged RH as a threshold, therefore conservatively overestimating risk. In order to produce a protocol for material performance following material failure, under failure's current definition, an accurate depiction of failure must be provided for risk assessment, beyond the standard RH limit relative to time.

\subsection{Aim and objective}

The aim of this paper is to contribute to the development of a more realistic depiction of interstitial mould growth risk assessment for more accurate risk prediction and material selection by formulating new criteria to assess materials based on published experimental data and evaluating their influence on the outcome of the risk assessment.

\section{Methodology}

\subsection{Description of data}

This study utilized secondary data from a subset of scenarios assessed in the 'Moisture Risk Assessment for Internal Wall Insulation in Solid Wall Buildings in Bristol' study [2]. Marincioni used WUFI Pro 5.3, for the parametric analysis of moisture and heat transfer in buildings in order to determine what might be considered as the best "generic" or "safe" solution to avoid mould risk for internal wall insulation systems, intentionally based on limited known local characteristics. Twenty-four different basic scenarios in four different insulation materials were simulated for 10 years, with the final year used for analysis [2].

\subsection{Proposed risk criteria}

The current methods of risk assessment are commonly based on assumptions of ideal environmental conditions for mould growth. The present investigation incorporated more specific critical $\mathrm{RH}$ values as presented by Sedlbauer [4,5] in addition to including the effect of material classification (relating nutrient availability) into a new set of criteria which could denote failure. This was accomplished by replacing the ADF's strict 75, 85, and 95\% critical RH limits tied to the duration of exposure, with new criteria to represent a spread of critical RH, varying with temperature and material classification (class $0=$ nutrient media, $1=$ readily biologically degradable, 2 = minimally biologically degradable), in addition to exposure time. The new criteria increased critical RH up to $15 \%$ from regulation standards (Table 1 ).

\subsection{Execution of analysis}

Temperature and RH data from Marincioni [2] were aligned in MS Excel and checked against the proposed criteria on a moving average basis, given the three durations (Table 1), in addition to the durations under the standard ADF criteria. 


\section{Results}

The analysis concerned the total number of hours exceeding the RH thresholds and the frequency of exceeding such thresholds on the subset of data, analyzed under both the ADF criteria and the new failure criteria. The results predicted 50-100\% less failure following analysis under the new criteria which accounted for temperature and material class (Table 2). All materials were assessed of class II, moderately resistant to fungal activity, but not completely resistan. Figure 1 demonstrates the potential differences in performance based on material class. Under current ADF criteria analysis, the wood fibre (WF) material presented the least frequency exceeding the threshold over the one-year timeline at $20 \%$, whereas MW-VCL, MW-AVCL, and PIR failed $~ 60-80 \%$ of the time.

Table 2. Initial results, comparing present ADF risk assessment standards to the newly proposed criteria, including hours of failure within test year as well as the frequency of exceeding the threshold. Frequency of exceeding the threshold was utilized to understand how often failure occurred and elucidate materials which may frequently exceed the threshold for short periods vs infrequently for substantial durations.

\begin{tabular}{|c|c|c|c|c|c|c|}
\hline \multirow[t]{2}{*}{ Material } & \multirow[t]{2}{*}{ Class } & \multicolumn{2}{|c|}{$\begin{array}{l}\text { Hours exceeding moving average } \\
\text { RH threshold over } 1 \text { month }\end{array}$} & \multicolumn{2}{|c|}{$\begin{array}{c}\text { Frequency of exceeding the } \\
\text { threshold }\end{array}$} & \multirow[t]{2}{*}{$\begin{array}{c}\% \text { Passing } \\
\text { (new criteria) }\end{array}$} \\
\hline & & ADF Criteria & New Criteria & ADF Criteria & New Criteria & \\
\hline $\begin{array}{c}\text { Mineral wool, vapor control layer } \\
\text { (MW-VCL) }\end{array}$ & II & 6779 & 3898 & 3 & 2 & 55.50 \\
\hline $\begin{array}{c}\text { Mineral wool, variable vapor control layer } \\
\text { (MW-AVCL) }\end{array}$ & II & 5141 & 0 & 2 & 0 & 100 \\
\hline $\begin{array}{c}\text { Polyisocyanurate } \\
(\mathrm{PIR})\end{array}$ & II & 6769 & 3901 & 2 & 2 & 55.47 \\
\hline $\begin{array}{l}\text { Capillary-active wood fibre } \\
\text { (WF) }\end{array}$ & II & 1818 & 0 & 1 & 0 & 100 \\
\hline
\end{tabular}

From this limited subset of data tested (one of 24 scenarios), the calculation for all materials returned fewer hours of predicted failure under the new criteria, with two materials returning no predicted failure and two materials undergoing approximately half as much predicted failure (58\%) as when calculated by ADF standards.

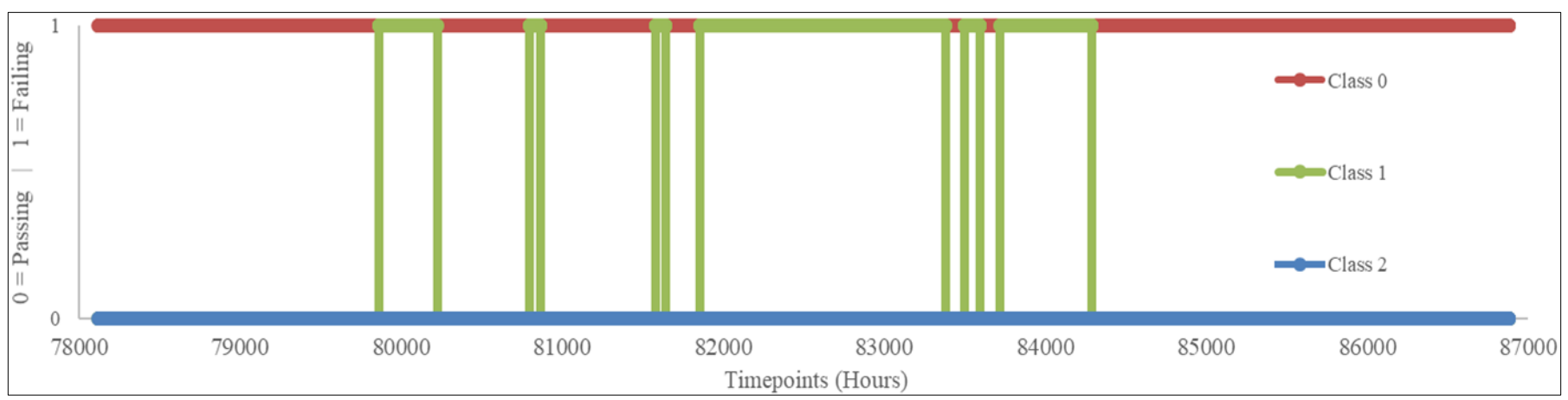

Figure 1 Representative example pass/fail of new criteria relative to time of observation for WF across 3 material classifications. Vertical lines representative of changes between passing and failing moving average $\mathrm{RH}$ limits.

\section{Discussion}

The more inclusive risk assessment criteria developed by this study to assess fungal risk within the interstitial space of IWI systems resulted in dramatically less failure overall. The augmented criteria incorporated temperature, which was accounted for by [2] following initial simulations in WUFI Pro 5.3, but not specifically denoted in the ADF standards. Additionally, nutrient availability of the substrate material (IWI in this case) was also included. Previously, this was not considered by the current government regulations (ADF) or standards. However, as mentioned previously, Sedlbauer [4,5] suggested that material classification should be considered when interpreting fungal activity risk, as nutrient availability directly affects the germination and growth rates of fungi. A test of materials as hypothetically class 0 or 1 (not class 2) increased failure ubiquitously suggesting that nutrient availability could, in fact, contribute a great deal to a more accurate depiction of mould risk and should be implemented into the present building regulations and guidelines.

\section{Conclusions}

This paper presented new criteria for the mould growth risk assessment of IWI at the wall-insulation interface. The criteria are easy to use, although more comprehensive than the currently used criteria. Upon further validation of the criteria proposed in this study for more realistic risk prediction, future revisions of the UK building regulations should consider adoption of such criteria and engage with literature which might provide a protocol for dealing with insulation materials post-failure.

\section{References}

[1] HM Government (2010) Approved Document F- Ventilation.

[2] Marincioni, V. (2015) Moisture Risk Assessment for Internal Wall Insulation in Solid Wall Buildings in Bristol, Technical Report, Sustainable Traditional Buildings Alliance (unpublished) 


\section{ICMB21}

[3] BSI (2011) BS 5250:2011 Code of practice for control of condensation in buildings. London: British Standards Institution

[4] Sedlbauer, K. (2001) Prediction of mould fungus formation on the surface of and inside building components.

[5] Sedlbauer, K. (2002) 'Prediction of Mould Growth by Hygrothermal Calculation', Journal of Thermal Envelope and Building Science. Sage Publications Sage CA: Thousand Oaks, CA, 25(4), pp. 321-336. doi: 10.1177/0075424202025004093. 